\title{
ESTUDO EXPERIMENTAL DO TIPO DE SEPARAÇÃO DE PARTÍCULAS EM UM HIDROCICLONE CONCENTRADOR
}

\author{
F. F. SALVADOR ${ }^{1}$, N. K. G. SILVA ${ }^{1}$, M. A. S. BARROZO ${ }^{1}$ e L. G. M. VIEIRA ${ }^{1}$ \\ ${ }^{1}$ Universidade Federal de Uberlândia, Faculdade de Engenharia Química \\ E-mail para contato: luizgustavo@feq.ufu.br
}

\begin{abstract}
RESUMO - Hidrociclones são separadores centrífugos que se destinam à separação de partículas sólidas ou líquidas de um fluido em função da densidade e distribuição granulométrica da fase dispersa. O objetivo deste trabalho foi estudar, experimentalmente, a separação no hidrociclone, a fim de se verificar se esta ocorria por diferença de densidade ou de tamanho das partículas. Para tanto, foi escolhido como objeto desse estudo um hidrociclone concentrador. Os ensaios experimentais foram conduzidos com suspensões aquosas de rocha fosfática em um nível de queda de pressão fixo e um picnômetro a gás hélio foi utilizado para determinação da densidade das partículas presentes nas correntes de alimentação, overflow e underflow. Diante dos resultados empíricos e posterior análise estatística, foi possível verificar, com $95 \%$ de confiabilidade, que não havia diferença entre as densidades das correntes do hidrociclone, concluindo que o equipamento realizava a separação pelo tamanho das partículas.
\end{abstract}

\section{INTRODUÇÃO}

Os hidrociclones são equipamentos versáteis, pois podem ser utilizados como separadores, tanto para suspensões diluídas quanto concentradas, sendo a fase dispersa sólida, líquida ou gasosa. Hidrociclones também são usados como clarificadores, concentradores e equipamento de lavagem, podendo ainda funcionar como desgaseificadores e classificadores (Heiskanen, 1993). Outras vantagens na utilização desse equipamento são a sua simplicidade de construção, o baixo custo de instalação e operação, a versatilidade de aplicação e seu pequeno tamanho quando comparado a outros separadores (Svarovsky, 2000).

Inicialmente os hidrociclones foram utilizados no beneficiamento de minérios, porém, a alta eficiência de separação e capacidade de processamento, aliadas ao pequeno espaço requerido por esse separador, os tornam ideais para operações onde o espaço físico é crítico. Atualmente este equipamento é empregado em diversos setores indutriais, tais como o petroquímico, alimentício, processamento mineral, extrativista etc (Svarovsky, 2000).

Hidrociclones são constituídos por uma seção cônica acoplada por uma seção cilíndrica na sua parte superior, com uma corrente de entrada e duas de saída (a diferentes concentrações). Entretanto, com o intuito de otimizar o desempenho desse separador, modificações vêm sendo realizadas em sua geometria. Existe uma classe de separadores denominados hidrociclones filtrantes, os quais foram desenvolvidos por pesquisadores da FEQUI/UFU, cuja diferença está na parede porosa da parte cônica (HCoF) em substituição à parede maciça. Tais separadores centrífugos proporcionam um menor consumo energético 
(número de Euler) nas mesmas condições operacionais de um hidrociclone totalmente maciço (Vieira et al., 2010).

Especificamente na separação sólido-líquido, a suspensão é injetada no equipamento de forma a proporcionar um vórtice externo, que por sua vez provoca um campo centrífugo responsável pela separação das fases. A suspensão diluída deixa o equipamento pelo topo do cilindro (overflow) e a suspensão concentrada deixa-o através de um orifício na base cônica do hidrociclone (underflow), como mostra a Figura 1.

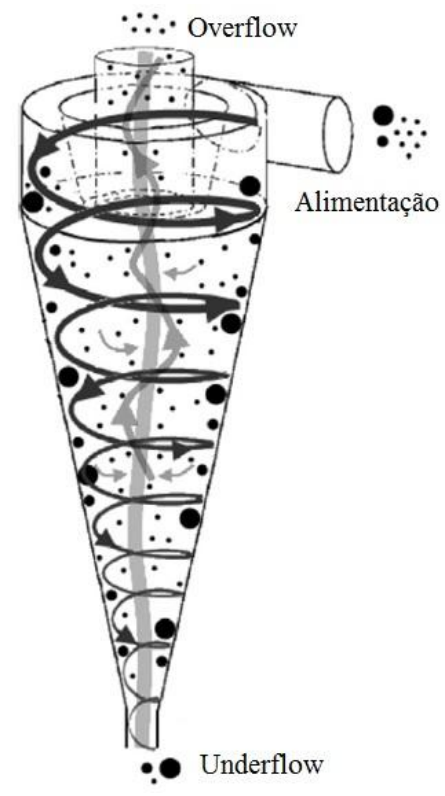

Figura 1 - Esquema do escoamento interno no hidrociclone (Adaptado de Davailles, 2012).

O princípio básico que promove a separação em hidrociclones é a sedimentação centrífuga: partículas suspensas sofrem ação da aceleração centrífuga, responsável por separálas do fluido. Segundo Svarovsky (2000), em tese, qualquer hidrociclone separa o sólido do líquido com base na diferença de densidade entre as fases, e o processo depende fortemente do tamanho da partícula, ou de sua densidade (se o sistema não for homogêneo).

Uma vez que o que o hidrociclone pode ser usado para separar partículas (suspensas em um líquido de baixa densidade) por tamanho, densidade ou velocidade terminal (Richardson e Harker, 2002), a caracterização do material particulado consiste em uma tarefa importante para determinar a natureza da separação.

O material particulado selecionado para este estudo foi o concentrado de rocha fosfática, que tem significativa importância para a região em torno do Município de Uberlândia (MG), localizado próximo a unidades produtoras de fertilizantes fosfatados. Em termos simples, o processamento desse material ocorre da seguinte forma: o minério de fosfato extraído da mina é destinado para beneficiamento a fim de produzir concentrado de $\mathrm{P}_{2} \mathrm{O}_{5}$ (pentóxido de difósforo), a partir do qual se formam os produtos finais (fertilizantes fosfatados, fosfato bicálcico para alimentação animal e ácidos para uso industrial, por exemplo). Conforme mostra a Tabela 1, o material particulado em estudo trata-se de um composto, o que suscita a dúvida de que se quando submetido à hidrociclonagem, sofre 
separação exclusivamente por diferença de tamanho entre partículas, ou também em virtude da diferença de densidade entre elas, o que é uma hipótese apropriada, haja vista as múltiplas substâncias que compõem o concentrado de rocha fosfática.

Tabela 1 - Composição química do concentrado de rocha de fosfática (Copebrás, 2013)

\begin{tabular}{|c|c|}
\hline Espécie & Composição química (\%) \\
\hline $\mathrm{CaO}$ & 47,65 \\
\hline $\mathrm{P}_{2} \mathrm{O}_{5}$ & 37,40 \\
\hline $\mathrm{Fe}_{2} \mathrm{O}_{3}$ & 2,42 \\
\hline $\mathrm{SiO}_{2}$ & 2,13 \\
\hline $\mathrm{Al}_{2} \mathrm{O}_{3}$ & 0,55 \\
\hline $\mathrm{BaO}$ & 0,53 \\
\hline $\mathrm{MgO}$ & 0,24 \\
\hline
\end{tabular}

Mainza et al. (2004), citam os efeitos negativos da separação por densidade em hidrociclones: partículas de baixa densidade são descarregas na corrente de overflow mesmo não sendo finas o bastante e partículas que seriam suficientemente finas para serem coletadas no overflow são descartadas no underflow por serem muito densas, o que prejudica os processos industriais que seguem a hidrociclonagem. Por isso os autores criaram uma alternativa, que consistiu numa modificação geométrica por meio da incorporação de um duto de overflow adicional.

Com base no exposto, o objetivo deste trabalho foi verificar experimentalmente se a separação de uma suspensão aquosa de concentrado de rocha fosfática por um hidrociclone filtrante concentrador ocorria devido somente à diferença de tamanho ou, se juntamente com a granulometria, haveria também o efeito da presença de partículas com diferentes densidades em função da liberação dos diversos minerais contidos nas partículas de concentrado fosfático por ação das forças cisalhantes durante a hidrociclonagem.

\section{MATERIAIS E MÉTODOS}

O hidrociclone selecionado para este estudo (HFOT2a) apresenta a seguinte configuração geométrica (dimensões em milímetros): $\mathrm{Dc}=30,0 ; \mathrm{Di}=6,3 ; \mathrm{Do}=8,1 ; \mathrm{Du}=$ 2,$5 ; \ell=12,0 ; \mathrm{L}=141,0 ; \mathrm{h}=61,0 ; \mathrm{H}=80,0$ e $\theta=17,8^{\circ}$ (Silva, 2014).

O hidrociclone filtrante apresentava um sistema de montagem modular e as peças se encaixavam por roscas ou travas mecânicas. Todas as peças eram de latão, exceto o duto de underflow, feito de Teflon, e o tronco de cone, constituído de bronze sinterizado. A espessura da parede, a porosidade e a permeabilidade do tronco de cone eram 2,5 mm, 11,51\% e 6,76 x $10^{-15} \mathrm{~m}^{2}$, respectivamente (Silva, 2014).

O concentrado de rocha fosfática foi o material particulado escolhido para ser utilizado neste trabalho por se tratar de um material de grande importância para a economia da região e foi fornecido pela Copebrás, localizada em Catalão (GO). A distribuição granulométrica típica da rocha fosfática utilizada no estudo foi obtida através do analisador de partículas por 
difração a laser (Oliveira et al., 2007; Santana et al. 2012). O modelo bi-paramétrico RosinRammler-Bennet (RRB), representado pela Equação 1, foi o que melhor se ajustou aos dados experimentais, com um coeficiente de correlação linear $(\mathrm{R})$ igual a 0,9910 , cujos parâmetros $\mathrm{d}_{63,2}$ e $\mathrm{n}$ foram $17,62 \mu \mathrm{m}$ e 0,76 , respectivamente.

$$
X=1-\exp \left[-\left(\frac{d_{p}}{d_{63,2}}\right)^{n}\right]
$$

A unidade experimental utilizada era constituída de um reservatório para armazenamento da suspensão, um agitador mecânico para homogeneizar a suspensão, uma bomba helicoidal que fornecia energia para o movimento da suspensão, um manômetro digital para indicar a queda de pressão entre a alimentação e duto de overflow do equipamento e um hidrociclone filtrante.

A suspensão utilizada durante os ensaios experimentais foi preparada de modo que sua concentração volumétrica de material particulado fosse 1\%. Para a coleta dos dados experimentais os testes foram realizados na queda de pressão fixa e igual a $147 \mathrm{kPa}$.

A densidade real do material particulado coletado em cada uma das correntes do hidrociclone foi deternimada através da técnica de picnometria com deslocamento de gás hélio, por um equipamento da Micromeritics modelo Accupyc 1330. Para se obter a densidade foram coletadas 5 amostras de suspensão de cada corrente (alimentação, overflow e underflow), que foram levadas a estufa por 42 horas à $105^{\circ} \mathrm{C}$, para posteriormente realizar a análise no picnômetro. $\mathrm{O}$ filtrado era isento de sólidos.

Em sequência, os resultados obtidos a partir do picnômetro foram analisados no software Statistica 7.1 através do qual se calcularam as densidades médias para as 3 correntes. Em seguida, foi realizada uma Análise de Variância (ANOVA). O objetivo desse teste estatístico foi determinar se as discrepâncias entre as médias dos tratamentos (neste caso, cada tratamento corresponde à densidade de uma corrente) eram maiores do que razoavelmente seria esperado da variação que ocorre dentro do tratamento (Box et al., 2005). Um teste de hipóteses está vinculado a essa análise e, no presente estudo, a hipótese de nulidade estabeleceu que as densidades das correntes do hidrociclone seriam iguais (hipótese de nulidade - Equação 2), enquanto a hipótese alternativa sustentaria o contrário (Hipótese Alternativa - Equação 3):

$$
\begin{aligned}
& H_{0}: \rho_{A}=\rho_{O}=\rho_{U} \\
& H_{1}: \rho_{A} \neq \rho_{O} \neq \rho_{U}
\end{aligned}
$$

Essa metodologia implica na tentativa de aceitação de um modelo aditivo, representado pela Equação 4, sendo necessário, então, verificar a adequabilidade desse modelo mediante análise dos resíduos. É suposto que os resíduos são variáveis aleatórias, independentes, com distribuição idêntica (normal) de média zero e variância constante.

$$
y_{t i}=\eta_{t}+\varepsilon_{t i}
$$




\section{RESULTADOS}

Os resultados obtidos para as densidades das correntes do hidrociclone filtrante (HFOT2a) através do picnômetro estão apresentados na Tabela 2.

Tabela 2 - Densidades das correntes de alimentação, overflow e underflow obtidos no picnômetro

\begin{tabular}{|c|c|c|c|}
\hline Amostra & $\boldsymbol{\rho}_{\mathbf{A}}\left(\mathbf{g} / \mathbf{c m}^{\mathbf{3}}\right)$ & $\boldsymbol{\rho}_{\mathbf{O}}\left(\mathbf{g} / \mathbf{c m}^{\mathbf{3}}\right)$ & $\boldsymbol{\rho}_{\mathbf{U}}\left(\mathbf{g} / \mathbf{c m}^{\mathbf{3}}\right)$ \\
\hline 1 & 3,2062 & 3,2078 & 3,2274 \\
\hline 2 & 3,2099 & 3,2089 & 3,2085 \\
\hline 3 & 3,2105 & 3,2081 & 3,2206 \\
\hline 4 & 3,1996 & 3,2098 & 3,2103 \\
\hline 5 & 3,2066 & 3,2078 & 3,2091 \\
\hline
\end{tabular}

A Tabela 3 apresenta os valores encontrados para as densidades médias de cada corrente e o respectivo desvio padrão.

Tabela 3 - Média e desvio padrão das densidades das correntes

\begin{tabular}{|c|c|c|}
\hline Corrente & $\boldsymbol{\rho}_{\mathbf{M}}\left(\mathbf{g} / \mathbf{c m}^{\mathbf{3}}\right)$ & $\boldsymbol{\sigma}_{\boldsymbol{\rho}}\left(\mathbf{g} / \mathbf{c m}^{\mathbf{3}}\right)$ \\
\hline Alimentação & 3,2066 & 0,0043 \\
\hline Overflow & 3,2085 & 0,0009 \\
\hline Underflow & 3,2152 & 0,0084 \\
\hline
\end{tabular}

Para realizar a análise dos dados construíu-se um quadro de ANOVA que está apresentado na Tabela 4 . O nível de significância utilizado no teste de hipótese foi 5\%.

Tabela 4 - Quadro de ANOVA para os dados da Tabela 2-

\begin{tabular}{|c|c|c|c|c|c|}
\hline $\begin{array}{c}\text { Fonte de } \\
\text { Variação }\end{array}$ & $\begin{array}{c}\text { Soma dos } \\
\text { Quadrados }\end{array}$ & $\begin{array}{c}\text { Graus de } \\
\text { Liberdade }\end{array}$ & $\begin{array}{c}\text { Quadrado } \\
\text { Médio }\end{array}$ & F & $\begin{array}{c}\text { Nível de } \\
\text { Significância - p }\end{array}$ \\
\hline Média & 154,5686 & 1 & 154,5686 & 5118899 & 0,000000 \\
\hline $\begin{array}{c}\text { Entre } \\
\text { Tratamentos }\end{array}$ & $2,048 \times 10^{-4}$ & 2 & $1,024 \times 10^{-4}$ & 3 & 0,06810 \\
\hline $\begin{array}{c}\text { Dentro do } \\
\text { Tratamento }\end{array}$ & $3,623 \times 10^{-4}$ & 12 & $0,302 \times 10^{-4}$ & & \\
\hline
\end{tabular}


Através da análise da Tabela 4, pode-se afirmar que existe confiabilidade estatística de 95\% para dizer que as densidades das correntes do hidrociclone filtrante HFOT2a são iguais (não há efeito de tratamento), de maneira que o teste de hipótese levou a aceitação de $\mathrm{H}_{0}$, pois o valor da probabilidade (p) calculada foi igual a 0,06810 que é maior do que o valor estabelecido para o nível de significância (fora da região de rejeição de $\mathrm{H}_{0}$ ).

Os valores observados para a densidade das correntes do hidrociclone em estudo e os resíduos do modelo aditivo (Equação 2) estão apresentados na Tabela 5.

Tabela 5 - Valores observados e resíduos para cada corrente

\begin{tabular}{|c|c|c|c|c|c|}
\hline \multicolumn{2}{|c|}{ Alimentação } & \multicolumn{2}{c|}{ Overflow } & \multicolumn{2}{c|}{ Underflow } \\
\hline $\begin{array}{c}\text { Valor } \\
\text { Observado }\end{array}$ & Resíduo & $\begin{array}{c}\text { Valor } \\
\text { Observado }\end{array}$ & Resíduo & $\begin{array}{c}\text { Valor } \\
\text { Observado }\end{array}$ & Resíduo \\
\hline 3,2062 & $-0,00036$ & 3,2078 & $-0,00068$ & 3,2274 & 0,01222 \\
\hline 3,2099 & 0,00334 & 3,2089 & 0,00042 & 3,2085 & $-0,00668$ \\
\hline 3,2105 & 0,00394 & 3,2081 & $-0,00038$ & 3,2206 & 0,00542 \\
\hline 3,1996 & $-0,00696$ & 3,2098 & 0,00132 & 3,2103 & $-0,00488$ \\
\hline 3,2066 & 0,00004 & 3,2078 & $-0,00068$ & 3,2091 & $-0,00608$ \\
\hline
\end{tabular}

A Figura 3 mostra o gráfico Resíduo versus Predito para o modelo aditivo. A partir desse gráfico verifica-se que o modelo é apropriado, pois os resíduos conjuntamente e individualmente para cada tipo de tratamento estão distribuídos aleatoriamente em torno de zero, indicando a variância constante para todos os valores de resposta.

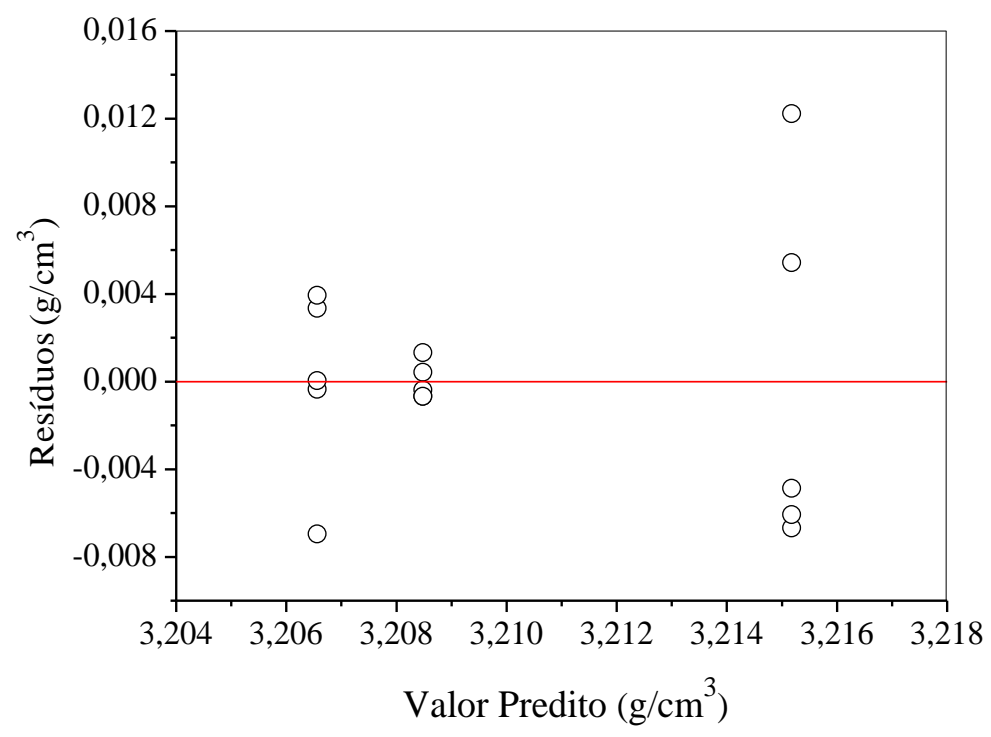

Figura 3 - Gráfico dos Resíduos versus Valor Predito. 


\section{CONCLUSÕES}

Este trabalho teve como objetivo verificar se a separação de uma suspensão aquosa de concentrado de rocha fosfática por um hidrociclone filtrante concentrador ocorria devido à diferença de tamanho ou de densidade entre as partículas dessa suspensão. De acordo com as condições operacionais e os principais resultados experimentais deste trabalho, foi possível constatar que as partículas do concentrado fosfático não foram fragmentadas durante o processo de hidrociclonagem, concluindo que a separação foi realizada apenas por diferença de tamanho do material. Através do resultado do teste de hipóteses verificou-se que não houve diferença significativa entre as densidades das correntes de concentrado e diluído do hidrociclone HFOT2a.

\section{NOMENCLATURA}

Dc - diâmetro do cilindro [mm]

Di -diâmetro do duto de alimentação [mm]

Do - diâmetro do overflow [mm]

$\mathrm{d}_{\mathrm{p}}$ - diâmetro da partícula [mm]

$\mathrm{Du}$ - diâmetro do underflow $[\mathrm{mm}]$

$\mathrm{d}_{63,2}$ - parâmetro do modelo RRB [mm]

$\mathrm{h}$ - altura da parte cilindríca [mm]

$\mathrm{H}$-altura do tronco de cone [mm]

$\ell$ - comprimento do vortex finder [mm]

$\mathrm{L}$ - comprimento total do hidrociclone [mm]

$\mathrm{n}$ - parâmetro do modelo RRB [-]

$\mathrm{X}$ - fração mássica de partículas iguais ou menores do que $\mathrm{d}_{\mathrm{p}}$ na corrente de alimentação [-]

$y_{t i}$ - i-ésimo valor observado na t-ésima coluna da tabela de dados $\left[\mathrm{g} . \mathrm{cm}^{-3}\right]$

$\varepsilon_{t i}$ - erro associado [g. $\mathrm{cm}^{-3}$ ]

$\eta_{t}-$ média do tratamento $\left[\mathrm{g} . \mathrm{cm}^{-3}\right]$

$\theta$-ângulo de abertura do tronco de cone $\left[^{\circ}\right]$

$\rho_{\mathrm{A}}-$ densidade das partículas da corrente de alimentação $\left[\mathrm{g} . \mathrm{cm}^{-3}\right]$

$\rho_{\mathrm{M}}$ - densidade média de cada corrente $\left[\mathrm{g} . \mathrm{cm}^{-3}\right]$

$\rho_{\mathrm{O}}-$ densidade das partículas da corrente de overflow $\left[\mathrm{g} . \mathrm{cm}^{-3}\right]$

$\rho_{U}-$ densidade das partículas da corrente de underflow $\left[\mathrm{g} \cdot \mathrm{cm}^{-3}\right]$

$\sigma_{\rho}-$ desvio padrão relacionado a densidade das correntes $\left[\mathrm{g} \cdot \mathrm{cm}^{-3}\right]$

\section{AGRADECIMENTOS}

Os autores agradecem à Fundação de Amparo à Pesquisa do Estado de Minas Gerais (FAPEMIG) pelos recursos concedidos no Projeto de Participação Coletiva em Eventos Técnicos-Científicos (PCE-00082-14) e ao Laboratório de Separação e Energias Renováveis (LASER) da FEQUI/UFU.

\section{REFERÊNCIAS}

BOX, G.E.P.; HUNTER, J.S.; HUNTER, W.G. Statistics for experimenters: design, 
innovation and discovery, 2nd ed., New Jersey: John Wiley and Sons, 2005.

COPEBRÁS (Companhia Petroquímica Brasileira). [S1.:sn], 2013.

DAVAILLES, A.; CLIMENT, E.; BOURGEOIS, F. Fundamental understanding of swirling flow pattern in hydrocyclones. Separ. Purif. Technol., v. 92, p. 152-160, 2012.

HEISKANEN, K. Particle Classification, London: Chapman \& Hall, 1993.

MAINZA, A.; POWELL, M.S.; KNOPJES, B. Differential classification of dense material in a three-product cyclone. Miner. Eng., v. 17, p. 573-579, 2004.

OLIVEIRA, M.S.; QUEIROZ, G.M.; GUIMARÃES, R.C.; ATAÍDE, C.H.; BARROZO, M.A.S. Selectivity in phosphate column flotation. Miner. Eng., v. 20, p. 197-199, 2007.

RICHARDSON, J. F.; HARKER, J. H. Particle technology and separation processes. 5th ed., v. 2. Oxford: Butterworth-Heinemann, 2002.

SANTANA, R.C.; RIBEIRO, J.A.; SANTOS, M.A.; REIS, A.S.; ATAÍDE, C.H.; BARROZO, M.A.S. Flotation of fine apatitic ore using microbubbles. Sep. Purif. Technol., v. 98, p. 402-409, 2012.

SILVA, N.K.G. Estudo de otimização da configuração do hidrociclone filtrante. Dissertação de Mestrado, Universidade Federal de Uberlândia (UFU), 2014.

SVAROVSKY, L. Solid-Liquid Separation. 4th ed., Oxford: Butterworth-Heinemann, 2000.

VIEIRA, L.G.M.; DAMASCENO, J.J.R.; BARROZO, M.A.S. Improvement of hydrocyclone separation performance by incorporating a conical filtering wall. Chem. Eng. Process, v.49, p.460-467, 2010. 